\title{
Association between Diabetes and Controlling Risk Factors with Survival Rate in Colorectal Cancer
}

\author{
Marjan Sharifi Nasab ${ }^{1,2}$ (D), Hamideh Yazdimoghaddam ${ }^{3,4 *(D)}$, Seyedeh Tahereh $\operatorname{Mohaddes}^{5}$ (D), \\ Mohammad Hassan Rakhshani6,7 iD
}

1. Dept. of Nursing, Faculty of Nursing and Midwifery, Sabzevar University of Medical Sciences, Sabzevar, Iran

2. Dept. of Nursing, Ghaem Hospital, Mashhad University of Medical Sciences, Mashhad, Iran

3. Dept. of Operating Room, Faculty of Paramedics, Sabzevar University of Medical Sciences, Sabzevar, Iran

4. Iranian Research Center on Healthy Aging, Sabzevar University of Medical Sciences, Sabzevar, Iran

5. Dept. of Hematology \& Medical Oncology, Ghaem Hospital, Faculty of Medicine, Mashhad University of Medical Sciences, Mashhad, Iran

6. Dept. of Biostatistics and Epidemiology, School of Public Health, Sabzevar University of Medical Sciences, Sabzevar, Iran

7. Iranian Research Center on Healthy Aging, Sabzevar University of Medical Sciences, Sabzevar, Iran

\begin{tabular}{|c|}
\hline Article Info \\
\hline doi $\underline{10.30699 / j a m b s .30 . e 56391}$ \\
\hline $\begin{array}{l}\text { Received: 2020/09/12; } \\
\text { Accepted: 2021/01/25; } \\
\text { Published Online: } 17 \text { Nov 2021; }\end{array}$ \\
\hline $\begin{array}{l}\text { Use your device to scan and read the } \\
\text { article online }\end{array}$ \\
\hline 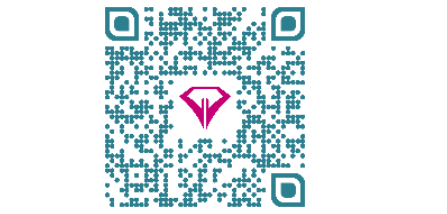 \\
\hline Article URL: Link \\
\hline $\begin{array}{l}\text { Corresponding Information: } \\
\text { Hamideh Yazdimoghaddam, } \\
\text { Dept. of Operating Room, Faculty of } \\
\text { Paramedics, Sabzevar University of } \\
\text { Medical Sciences, Sabzevar, Iran. }\end{array}$ \\
\hline $\begin{array}{l}\text { E-Mail: } \\
\text { ha_yazdimoghaddam@yahoo.com }\end{array}$ \\
\hline
\end{tabular}

\begin{abstract}
Background \& Objective: Colorectal cancer (CRC) is a highly prevalent malignancy leading to death across the world. However, patient survival is greatly affected following a diagnosis of diabetes. The purpose of this research was to study the correlation between diabetes and controlling risk factors with the survival rate of colorectal cancer patients.
\end{abstract}

Materials \& Methods: This retrospective follow-up study was done on 356 patients with colorectal cancer, who had been diagnosed during 1999-2013. Notably, the patients were selected using systematic random sampling. The patients were allocated into two groups according to the history of diabetes and its absence. The survival rate of the patients was assessed during the follow-up period up to 2018 .

Results: In colorectal cancer patients, the diabetes prevalence was $11 \%$. The mean survival rate in patients with CRC and non-colorectal non-diabetic was 5.541 \pm 0.227 years and in diabetic patients was $0.508 \pm 5.246$ years. No significant difference was apperceived in the survival rate of the diabetic and non-diabetic patients $(\mathrm{P}=0.57)$. The risk of mortality in patients with CRC who were not treated with insulin increased and their survival decreased $(\mathrm{P}=0.05)$. In metastatic stage of lymph nodes, it was 0.314 higher in patients with the metastasis to distant regions $(\mathrm{P}=0.000)$.

Conclusion: Based on the results, training diabetic patients regarding effective therapies in controlling risk factors, hyperglycemia, and screening for rapid diagnosis of CRC are essential to improve health and increase survival rate in diabetic patients.

Keywords: Diabetes Mellitus, Insulin, Survival, Colorectal Neoplasms, Neoplasm Metastasis

\section{Introduction}

Today, cancer is a main public health threat across the world especially in developing countries (1). Colorectal cancer (CRC) is the third most common cancer and affecting 1.23 million persons $(9.7 \%$ of all cancer types) annually (2). CRC is the third leading cause of death in the United States (3). CRC is the fourth most common cancer in men and the second most common in women in Iran. Recent researches have indicated rapid progress in the incidence of CRC in Iran (2017). As well, CRC was reported as the fifth cause of mortality in males and the fourth in females (4).
CRC is the most common gastrointestinal cancer, and its outbreak has increased over the last 25 years, and Iranian data show younger age distribution than Western reports (5). Overall, CRC is ranked third in incidence and second in mortality rates. CRC incidence rates vary widely (6). The statistic of new cancer cases is anticipated to increase, and cancers causing death are also predicted to reach over 11.4 million in 2030 across the world, manifesting cancers to be the major cause of death (7).

Based on the scientific reports, Out of $40 \%$ of cancers could be prevented by decreasing the risk 
factors (8). The correlation between type II diabetes and some cancers has been identified, while this diagnostic complexity is remarkably increasing. Some types of cancer, especially CRC are affected by some risk factors, including diabetes, insulin resistance, age, gender, socioeconomic status, obesity, insulin resistance, diet, alcohol consumption, physical activity, and smoking habits (9). The global prevalence of diabetes is a risk factor for more seriously considering the disease (10). In addition, some research studies have investigated to identify the mechanisms of how diabetes and cancer are associated together (11). In the study by Fedeli et al. (2014), different types of biological pathways, such as hyperinsulinemia, hyperglycemia, and persistent antiinflammatory status have been reported as risk factors for cancer in diabetes (12).

There is an association between type 2 diabetes and increased colon, breast, pancreatic, and other cancers. The risk of cancer in diabetic patients increases because of the rising prevalence of diabetes, the extensive use of insulin analogs, as well as other anti-diabetic drugs, which gives presuppositions regarding cancer risk in the diabetic population (13).

Recently, some factors have also been identified to increase the risk of cancer, such as insulin glargine. It has raised concerns about the increasing use of insulin; since studies have shown that insulin is related to a raised risk of cancer (14), while metformin appears to be related to a decreased risk of cancer and mortality (15).

Moreover, diabetic patients were reported to have a poor prognosis after cancer diagnosis as well as increasing cancer risk (10).

Evidence has shown an increased mortality rate caused by colorectal, breast, endometrial, kidney, liver, and pancreatic in patients with diabetes. Nevertheless, it remains unclear whether or not diabetes is an independent risk factor for the mentioned neoplasms $(10,16)$.

On the other hand, several reasons have been shown to decrease survival in diabetic patients after diagnosis of cancer (10), including increased tumor cell proliferation due to hyperinsulinemia and hyperglycemia, different methods of cancer treatment, poor response to cancer therapy in diabetic patients, as well as inadequate diabetes management during cancer treatment. Though, these data have been poorly evaluated and require further investigation.

Despite receiving multiple treatments, the survival rate of these patients declines due to the metastatic nature of cancer (9). However, the diagnosis of cancer patients with diabetes increases the complications in the treatment process, which in turn reduces the survival rate. Therefore, the purpose of this research was to study the correlations between diabetes and controlling risk factors, such as smoking, obesity, insulin resistance, and drug therapy with the five-year survival rate in CRC patients.

\section{Materials and Methods}

This retrospective follow-up study was conducted to analyze the patients' data diagnosed with $\mathrm{CRC}$ receiving treatment at clinics affiliated with Mashhad University of Medical Sciences.

\section{Participants}

The research populations were patients with diagnosed CRC from 1999 to 2013 referred to the oncology department of Imam Reza and Ghaem Hospital, and also Isar Chemotherapy Center.

The inclusion criteria of the study were the patients with a pathological diagnosis of colorectal carcinoma and age of more than 18 years. On the other hand, the exclusion criteria consisted of family history of CRC, incomplete treatment of cancer due to leaving the hospital with personal consent, and failure to complete the treatment. After receiving approvals from the Ethics Committee of the university, the medical records related to the $1999-2013$ period were selected by systematic random sampling, and the related variables were systematically extracted.

Demographic and clinical information of patients were collected through a questionnaire accurately to assess risk factors and history of diabetes.

Overall, the following demographic and clinical data were collected for medical records of 356 desirable patients. Then the patients were divided into two groups based on the history of diabetes and its absence. The patients diagnosed with CRC were followed up to evaluate survival time up to five years since the diagnosis to investigate the relationship between diabetes and risk factors with survival rate.

The mean follow-up period was $5.0 \pm 0.1$ years.

CRC diagnosis was confirmed based on the criteria of the International Classification of Pathologies and Diseases (ICD), the American Commission on Cancer, and the pathology report. To make a diabetes mellitus (DM) diagnosis, fasting blood sugar (FBS) and $\mathrm{HbA}_{1} \mathrm{c}$ tests were precisely carried out. Diagnosis or detection of diabetes (FBS levels $\geq 126 \mathrm{mg} / \mathrm{dl}$ ) at recurrence was recorded.

\section{Statistical Analysis}

Data analysis was fulfilled in SPSS version 17 (Chicago, IL, USA) using descriptive statistics, t-test or its nonparametric equation, Mann-Whitney U test, Chisquare, and Cox proportional hazards model to assess risk factors and hazard ratio (HR) in the diabetic and nondiabetic CRC patients. In all the statistical analysis, the Pvalue of less than 0.05 was considered significant.

\section{Ethical consideration}

This research was approved by the Sabzevar University of Medical Sciences (Ref : 97081 ) and Ethics Committee of Sabzevar University of Medical Sciences, sabzevar, Iran (ethical code: IR.MEDSAB.REC.1397.020). 


\section{Results}

The mean age of the diabetic and non-diabetic CRC patients was 65.00(12.61) and 55.47(15.05), respectively. The demographic characteristics of the participants consisted of gender, marital status, residence, and job status. The results of the Fisher's exact test and Chi-square test demonstrated no significant differences between diabetic and nondiabetic patients $(\mathrm{p}>0.05)$.

The mean duration of diabetes in patients with CRC was $10.38 \pm 7.38$. Evaluation of cancer risk factors in the two diabetic and non-diabetic groups indicated that $97.4 \%$ of the diabetic patients and $95.0 \%$ of non-diabetic patients had negative smoking habits. The results of Fisher's exact test demonstrated no significant differences between diabetic and non-diabetic patients $(\mathrm{P}=0.58)$.

In addition, other risk factors, such as the Body Mass Index (BMI), mean and standard deviation of in the diabetic group was 25.66 (2.75) and in the non-diabetic group was 24.56 (4.64). Mann-Whitney test demonstrated a significant difference between the two groups $(\mathrm{P}=0.04)$.

According to the histopathological diagnosis, 51.3\% of diabetic patients and $46.1 \%$ of non-diabetic patients were in the non-metastatic stage. However, Chi-square results demonstrated no significant difference between diabetic and non-diabetic patients $(\mathrm{P}=0.51)$. The results related to tumor markers (e.g., CEA and CA19-9 in CRC) are presented in Table 1.

Table 1: Frequency distribution of tumor markers for patients with CRC

\begin{tabular}{cccc}
\multirow{2}{*}{ Tumor Markers } & Patient & Mean (SD) & P- Value \\
\hline \multirow{2}{*}{ CEA } & Diabetic & $20.01(28.75)$ & 0.03 \\
& Non Diabetic & $58.94(314.12)$ & 0.23 \\
\hline \multirow{2}{*}{ CA19-9 } & Diabetic & $59.87(115.18)$ & $141.18(913.19)$ \\
\hline
\end{tabular}

Based on the findings, the prevalence of diabetes was $11 \%$ in CRC patients, and the mean duration of diabetes was $10.38 \pm 7.38$ years. About chronic complications after diabetes, $59.0 \%$ of diabetic patients with CRC had no chronic complications after diabetes and $33.3 \%$ had hypertension.

Concerning controlling risk factors, such as diabetes controlling drugs; $87.2 \%$ of the patients used metformin and glibenclamide; while $7.7 \%$ of the subjects consumed metformin. Moreover, $79.5 \%$ of the patients had no history of insulin therapy, and the history of insulin therapy in diabetic patients was 5 (2.53) years.

The mean survival rate of non-diabetic and diabetic patients with CRC was 5.541 \pm 0.227 and $5.246 \pm 0.508$ years, respectively.

To investigate the impacts of diabetes on the survival rate of CRC patients in the presence of effective factors, such as age, gender, smoking, BMI, residence, marital status, occupation, tumor stage, and CEA tumor marker, the related data were fitted in the Cox regression model. However, the findings indicated no significant difference between survival time of diabetic and non-diabetic patients (95\% CI: $0.709-1.860$; HR: $1.149 ; \mathrm{P}=0.57)$.

Based on the above-mentioned risk factors, for the tumor stage, the mortality risk significantly decreased in patients with the non-metastatic stage (0.180 equal) compared to other patients and in the metastatic stage of lymph nodes ( 0.314 equal) compared to the patients with metastasis to distant regions, and their survival rate increased.

Analysis of the Cox regression model for diabetes control drugs demonstrated that the risk of mortality increased in CRC patients who were not treated with insulin (2.568 equal) and their survival rate decreased (95\% CI: 1.023 - 6.447; HR: 2.568; P = 0.05). In CRC cases, the effects of risk factors and using drugs on the survival rate of diabetic and non-diabetic patients are presented in Table 2 .

\section{Table 2: The Effect of Diabetes, Risk Factors, and Drugs on the Survival Rate of Patients with CRC}

\begin{tabular}{cccc} 
Variable & HR & $\mathbf{9 5 \%}$ CI & P- Value \\
\hline diabetes & 1.149 & $0.709-1.860$ & 0.57 \\
\hline non-metastatic stage & 0.180 & $0.122-0.266$ & 0.000 \\
\hline lymph node metastasis & 0.314 & $0.205-0.483$ & 0.000 \\
\hline insulin & 2.568 & $1.023-6.447$ & 0.05 \\
\hline
\end{tabular}




\section{Discussion}

According to the overview of CRC survival rates in Asia, mortality rates remain high (17). Recent evidence has suggested that CRC is the fifth cause of mortality in men and the fourth in women (4). In the current study, no significant difference was discovered between diabetic and non-diabetic groups in terms of the survival rate, which is consistent with the study by Karlin et al. (18). Therefore, it seems that diabetes does not independently affect the survival rate. Though mortality in the diabetic patients was over than that of the non-diabetic patients and the mean survival was reduced. Therefore, the impact of diabetes as a risk factor on cancer deaths cannot be ignored. In another study, De Bruijn et al. (2013) considered diabetes as a risk factor for CRC and cancer death (19). In similar research, Rosato et al. also found a positive association between diabetes and CRC (20). This discrepancy between the present study findings and the aforementioned studies might be due to metformin use in diabetic patients with CRC, which increased the survival rate of the subjects. In summary, further investigation is recommended to confirm the present study findings.

History evaluation based on anti-diabetic drugs revealed a significant decrease in the survival rate of patients who were not treated with insulin. In a study by Rosato et al. a positive association was found between insulin use and CRC (20). Moreover, Dąbrowski et al. reported that $\mathrm{HbA} 1 \mathrm{c} \geq 8.5 \%$, insulin treatment in a dosedependent manner, and time-varying method, had a significant association with a raised risk of malignancy (21). Also, a study by Call et al. has shown that the rate of tumor growth progression in the presence of insulin analogs has been increased. However, the positive effects of insulin therapy on decreasing diabetes-related deaths were greater than these risks (14). Hyperglycemia directly or indirectly causes DNA damage, DNA breaks, mutation accumulation, and impairment in DNA repair, by changing the expression of oncogenes and tumor suppressor genes using various mechanisms (22). Induced mutations in oncogenes and tumor suppressor genes stimulate cancer cell proliferation, which can reduce the survival rate of diabetic patients with CRC (11).

Therefore, physicians should regularly screen cancer patients receiving insulin and monitor tumor growth or disease recovery (14). Lack of proper control of chronic hyperglycemia in diabetes is associated with serious clinical consequences, leading to long-term complications, such as retinopathy, peripheral neuropathy, nephropathy, peripheral vascular disease, ischemic heart disease, stroke, limb complications, and infections (22).

The results of the present study revealed a significant decrease in survival time in CRC patients with distant metastasis. Ahmadi et al. have reported that the incidence of death in the non-metastatic and metastatic CRC with lymph node involvement was lower than patients with distant metastasis (23); since in the non-metastatic stage, there was a better response to cancer treatment. However, in the metastatic phase, the response to treatment is poor. The results of the systematic review and meta-analysis by
Tahmasbi et al. have revealed a relationship between tumor metastasis and the survival rate of the patients in a way that the mortality risk of patients with metastasis who were referred to other centers was 3.26 times more than those without it (24). A study by Akhoond et al. has reported that the mortality risk of individuals with tumor metastasis who were referred to other centers was 3.577 for colon cancer and 2.646 for rectum cancer compared to those without it (25).

The evaluation of risk factors revealed that the BMI of diabetic patients with $\mathrm{CRC}$ was higher than nondiabetic patients. Erickson et al. concluded that the overall risk of mortality was twice higher in the women with the HbA1C level of $\geq \% 7.0$ after modifying the confounding factors compared to the subjects with HbA1C level less than $6.5 \%$ (26), which might be due to the reduced susceptibility to anti-angiogenic regimens (27). Obesity raises the prevalence of CRC and affects outcomes, overall and in association with specific CRC treatments (28). Murphy et al. reported that obesity increases the risk of colon cancer mortality especially among males (29). Due to the importance of metabolic syndrome and insulin resistance, BMI was recognized as a risk factor in the diagnosis of diabetic patients with CRC. Despite different results, training diabetic patients regarding risk factors of CRC, such as BMI, and effective therapies in controlling hyperglycemia, such as insulin, and also CRC screening in the early stages of CRC are essential for the improvement of diabetic patients health and increasing their survival rate.

\section{Conclusion}

The high prevalence of CRC is an important public health issue. According to the study results, diabetic patients are at risk of developing types of cancer, especially CRC due to drug treatments and the presence of risk factors. Therefore, screening is important.

Due to the increased survival in insulin-treated patients, the treatment of hyperglycemia in diabetic patients with CRC is important. The reduced risk of death in patients with non-metastatic and metastatic CRC with Lymph node involvement suggests the importance of secondlevel prevention in the timely treatment of CRC.

Therefore, early screening of diabetic patients by physicians, for faster diagnosis of CRC in early stages and timely treatment and prevention of metastasis can improve their survival.

According to the results of the study, in the followup survival of patients with CRC and diabetes, due to the increased risk of death in patients with metastasis to distant sites, Identification of risk factors, early diagnosis, and education of diabetic patients regarding control of hyperglycemia with effective therapies could help increase the survival of diabetic patients with CRC. 


\section{Acknowledgment}

This article is derivative from the Master's thesis in nursing approved by Sabzevar University of Medical Sciences with code 97081 . Hereby, thanks are owed to the authorities of the hospitals affiliated with Mashhad University of Medical Sciences for their assistance in conducting this research project.

\section{Conflicts of Interest}

There is no conflict of interest.

\section{References}

1. Malvezzi M, Carioli G, Bertuccio P, et al. European cancer mortality predictions for the year 2018 with focus on colorectal cancer. Ann Oncol. 2018 29(4):1016-22. [DOI:10.1093/annonc/mdy033] [PMID]

2. Dolatkhah R, Somi MH, Kermani IA, et al. Increased colorectal cancer incidence in Iran: a systematic review and meta-analysis. BMC public health. 2015 15(1):997. [DOI:10.1186/s12889015-2342-9] [PMID] [PMCID]

3. Kasper DL, Hauser SL, Fauci AS, et al. Harrisons internal medicine. 19th, editor. United States of America: MCGraw-Hill; 2015.

4. Organization WH. Country Cancer Profile. Available from: http://www who int/cancer/country-profiles/chn_en pdf. 2014 [Accessed 5 may 2021].

5. Pourhoseingholi MA, Zali MR. Colorectal cancer screening: Time for action in Iran. World J Gastrointest Oncol. 2012;4(4):82-3. [DOI:10.4251/wjgo.v4.i4.82] [PMID] [PMCID]

6. Bray F, Ferlay J, Soerjomataram I, et al. Global cancer statistics 2018: GLOBOCAN estimates of incidence and mortality worldwide for 36 cancers in 185 countries. CA Cancer J Clin. 2018;68(6):394-424. [DOI:10.3322/caac.21492] [PMID]

7. Bray F, Jemal A, Grey N, et al. Global cancer transitions according to the Human Development Index (2008-2030): a population-based study. Lancet Oncol. 2012;13(8):790-801. [DOI:10.1016/S1470-2045(12)70211-5]

8. Jemal A, Center MM, DeSantis C, et al. Global patterns of cancer incidence and mortality rates and trends. Cancer Epidemiol Biomarkers Prev. 2010 19(8):1893-907. [DOI:10.1158/1055-9965.EPI10-0437] [PMID]

9. Wild S. Diabetes, treatments for diabetes and their effect on cancer incidence and mortality: attempts to disentangle the web of associations. Diabetologia. 2011;54(7):1589-92. [DOI:10.1007/s00125-011-2169-6] [PMID]
10. Onitilo AA, Engel JM, Glurich I, et al. Diabetes and cancer I: risk, survival, and implications for screening. Cancer Causes Control. 2012;23(6):967-81. [DOI:10.1007/s10552-0129972-3] [PMID] [PMCID]

11. Collins KK. The diabetes-cancer link. Diabetes Spectr. 2014;27(4):276-80.

[DOI:10.2337/diaspect.27.4.276]

[PMID] [PMCID]

12. Fedeli U, Zoppini G, Gennaro N, et al. Diabetes and cancer mortality: a multifaceted association. Diabetes research and clinical practice. 2014;106(3):e86-e9. [DOI:10.1016/i.diabres.2014.09.027] [PMID]

13. Pandey A, Forte V, Abdallah M, et al. Diabetes mellitus and the risk of cancer. Minerva endocrinologica. 2011;36(3):187-209.

14. Call R, Grimsley M, Cadwallader L, et al. Insulincarcinogen or mitogen? Preclinical and clinical evidence from prostate, breast, pancreatic, and colorectal cancer research. Postgrad Med. 2010;122(3):158-65. [DOI:10.3810/pgm.2010.05.2153] [PMID]

15. Lee JH, Kim TI, Jeon SM, et al. The effects of metformin on the survival of colorectal cancer patients with diabetes mellitus. Int $\mathbf{J}$ Cancer. 2012;131(3):752-9. [DOI:10.1002/ijc.26421] [PMID]

16. Behrouzi B, Mohagheghi MA, Sadighi S. Demographic Characteristics, Survival and Prognostic Factors of Early Breast Cancer Patients with Type 2 Diabetes Mellitus: A Hospital-Based Cohort Study. APJCP. 2017; 18(9):2485.

17. Moghimi-Dehkordi B, Safaee A. An overview of colorectal cancer survival rates and prognosis in Asia. World J Gastrointest Oncol. 2012;4(4):71. [DOI:10.4251/wjgo.v4.i4.71] [PMID] [PMCID]

18. Karlin NJ, Amin SB, Kosiorek HE, et al. Survival and glycemic control in patients with colorectal cancer and diabetes mellitus. Future Sci OA. 2018; 4(9):FSO335. [DOI:10.4155/fsoa-2018-0044] [PMID] [PMCID]

19. De Bruijn K, Arends L, Hansen B, et al. Systematic review and meta-analysis of the association between diabetes mellitus and incidence and mortality in breast and colorectal cancer. Br J Surg. 2013;100(11):1421-9. [DOI:10.1002/bjs.9229] [PMID]

20. Rosato V, Tavani A, Gracia-Lavedan E, et al. Type 2 diabetes, antidiabetic medications, and colorectal cancer risk: two case-control studies from Italy and Spain. Front Oncol. 2016;6:210. [DOI:10.3389/fonc.2016.00210] [PMCID]

[PMID] 
21. Dąbrowski M, Szymańska-Garbacz E, Miszczyszyn Z, et al. Risk factors for cancer development in type 2 diabetes: A retrospective case-control study. BMC cancer. 2016;16(1):785. [DOI:10.1186/s12885-016-2836-6] [PMID] [PMCID]

22. Ramteke P, Deb A, Shepal V, et al. Hyperglycemia associated metabolic and molecular alterations in cancer risk, progression, treatment, and mortality. Cancers. 2019; 11(9):1402. [DOI:10.3390/cancers11091402]

[PMID] [PMCID]

23. Ahmadi A, Mobasheri M, Hashemi Nazari SS. Survival time and relative risk of death in patients with colorectal cancer in an Iranian population: a cohort study. J Mazandaran Univ Med Sci. 2014;24(111):2-8.

24. Tahmasbi B, Abedi G, Moosazadeh M, et al. Determining the survival rate of colorectal cancer in Iran: A systematic review and meta-analysis. Asian Pacific journal of cancer prevention: APJCP. 2018; 19(11):3009.

[DOI:10.31557/APJCP.2018.19.11.3009] [PMID] [PMCID]
25. Akhoond MR, Kazemnejad A, Hajizadeh E, et al. Comparison of colon and rectum cancer:survival and prognostic factors. Gastroenterol Hepatol Bed Bench. 2010;3:177-86.

26. Erickson K, Patterson RE, Flatt SW, et al. Clinically defined type 2 diabetes mellitus and prognosis in early-stage breast cancer. J Clin Oncol. 2011;29(1):54-60.

[DOI:10.1200/JCO.2010.29.3183] [ [PMID] [PMCID]

27. Forouhi NG, Wareham NJ. Epidemiology of diabetes. Medicine. 2010;38(11):602-6. [DOI:10.1016/j.mpmed.2010.08.007]

28. Bardou M, Barkun AN, Martel M. Obesity and colorectal cancer. Gut. 2013;62(6):933-47. [DOI:10.1136/gutjnl-2013-304701] [PMID]

29. Murphy TK, Calle EE, Rodriguez C, et al. Body mass index and colon cancer mortality in a large prospective study. Am J Epidemiol. 2000;152:84754. [DOI:10.1093/aje/152.9.847] [PMID]

30.

\section{How to Cite This Article:}

Sharifi Nasab M, Yazdimoghaddam H, Mohaddes S T, Rakhshani M H. Association between Diabetes and Controlling Risk Factors with Survival Rate in Colorectal Cancer. J Adv Med Biomed Res. 2022; 30 (138) :2429

\section{Download citation:}

$\underline{\text { BibTeX }}|\underline{\text { RIS }}| \underline{\text { EndNote }}|\underline{\text { Medlars }}| \underline{\text { ProCite }}|\underline{\text { Reference Manager }}| \underline{\text { RefWorks }}$ 